\title{
Contingency awareness in human aversive conditioning involves the middle frontal gyrus
}

\author{
Ronald McKell Carter, ${ }^{\mathrm{a}, *}$ John P. O’Doherty, ${ }^{\mathrm{b}, \mathrm{c}}$ Ben Seymour, \\ Christof Koch, ${ }^{\mathrm{d}}$ and Raymond J. Dolan ${ }^{\mathrm{b}}$ \\ ${ }^{a}$ MC216-76 Division of Biology, California Institute of Technology, Pasadena, CA 91125, USA \\ ${ }^{\mathrm{b}}$ Wellcome Department of Imaging Neuroscience, 12 Queen Square, London, WC1N 3BG, UK \\ ${ }^{\mathrm{c}}$ Humanities and Social Sciences, California Institute of Technology, Pasadena, CA 91125, USA \\ ${ }^{\mathrm{d}}$ Computation and Neural Systems, California Institute of Technology, Pasadena, CA 91125, USA
}

Received 26 April 2005; revised 19 July 2005; accepted 1 September 2005

Available online 24 October 2005

\begin{abstract}
In contrast to the wealth of data describing the neural mechanisms underlying classical conditioning, we know remarkably little about the mechanisms involved in acquisition of explicit contingency awareness. Subjects variably acquire contingency awareness in classical conditioning paradigms, in which they are able to describe the temporal relationship between a conditioned cue and its outcome. Previous studies have implicated the hippocampus and prefrontal cortex in the acquisition of explicit knowledge, although their specific roles remain unclear. We used functional magnetic resonance imaging to track the trial-by-trial acquisition of explicit knowledge in a concurrent trace and delay conditioning paradigm. We show that activity in bilateral middle frontal gyrus and parahippocampal gyrus correlates with the accuracy of explicit contingency awareness on each trial. In contrast, amygdala activation correlates with conditioned responses indexed by skin conductance responses (SCRs). These results demonstrate that brain regions known to be involved in other aspects of learning and memory also play a specific role, reflecting on each trial the acquisition and representation of contingency awareness.

(C) 2005 Elsevier Inc. All rights reserved.
\end{abstract}

\section{Introduction}

Learning about aversive stimuli in the environment is necessary for an organism's success. One of the simplest and best studied mechanisms by which this is realized is classical conditioning, whereby a predictive association is learned between a neutral stimulus (the conditioned stimulus or CS) and a biologically meaningful signal (the unconditioned stimulus or US) (Pavlov, 1906; Cook and Harris, 1937; Wilensky et al., 1999; Buchel and Dolan, 2000; Maren, 2001; Clark et al., 2002; LeDoux, 2003; Maren and Quirk, 2004). Typically, after repeated pairings of CS

\footnotetext{
* Corresponding author. Fax: +1 6267968876.

E-mail address: mckell@klab.caltech.edu (R.M. Carter).

Available online on ScienceDirect (www.sciencedirect.com).
}

and US, the CS comes to elicit a response that is appropriate to the anticipated US. In aversive conditioning, this conditioned response (CR) will often be a change in heart rate or skin conductance, and is taken as an implicit measure of successful conditioning in experimental studies.

It is possible to become consciously aware of the predictive contingency between CS and US, a phenomenon referred to as contingency awareness. An individual can acquire both implicit associations and contingency awareness or either may be acquired independently (Bechara et al., 1995) indicating some degree of dissociation between the two systems. Currently, a major question in conditioning (and consciousness) research is the extent, and mechanism, of contingency awareness effects in conditioning (Hilgard et al., 1937; Cole, 1939; Dawson and Furedy, 1976; Lovibond and Shanks, 2002; Wiens and Ohman, 2002; Olsson and Phelps, 2004). A better understanding of contingency awareness and how it can facilitate or inhibit implicit associations is critical for a rational treatment of phobias, placebo effects, and anxiety disorders (Grillon, 2002; Quirk and Gehlert, 2003; Colloca and Benedetti, 2005).

The acquisition of contingency awareness and its interaction with conditioning differ across conditioning protocols (Clark and Squire, 1998; Ohman and Soares, 1998; Knuttinen et al., 2001; Han et al., 2003). For instance, in trace conditioning, in which there is temporal separation between CS and US, contingency awareness has been shown to correlate positively with the amplitude of conditioned responses(Clark and Squire, 1998). Those subjects in a trace conditioning experiment who do not display contingency knowledge fail to be trace conditioned. By contrast, in delay conditioning, in which there is no separation between the CS and US, no correlation between contingency awareness and successful conditioning has been observed; either can be acquired in the absence of the other. However, the simplicity of the delay protocol often results in immediate acquisition of explicit knowledge, making separation of explicit from implicit processes using a delay paradigm alone difficult. 
In this study, we used functional magnetic resonance imaging (fMRI) to identify brain regions that were specifically related to the explicit acquisition of contingency awareness during both delay and trace conditioning, independent of individual protocols. We simultaneously conditioned human subjects to predict an aversive electrical stimulus (US) from arbitrary visual cues (CS) with concurrent delay and trace protocols (see Figs. 1a, b). The use of simultaneous conditioning allowed us to identify brain responses specifically correlated with contingency awareness and distinct from responses associated with measures of implicit knowledge. To assess contingency awareness, subjects reported their shock expectancy on each trial (Figs. 1c, d), and in addition filled out a post-experimental questionnaire. These measures were then used to identify brain responses that correlated with accurate contingency awareness. We predicted that activity in dorsolateral prefrontal cortex and hippocampus would correlate with these measures of explicit knowledge based on evidence that these structures are involved in working memory (Leung et al., 2002), memory formation (Fanselow, 2000), and revaluation (Corlett et al., 2004), as well as from lesion studies of trace conditioning deficits (Compton et al., 1997; Clark and Squire, 1998; Kronforst-Collins and Disterhoft, 1998; McEchron et al., 1998).

\section{Methods}

\section{Participants}

We recruited 16 healthy right-handed subjects. Two were excluded: one because of excessive movement-related artifact precluding image analysis, and another subject who did not have at least one significant skin conductance response (SCR) for each trial
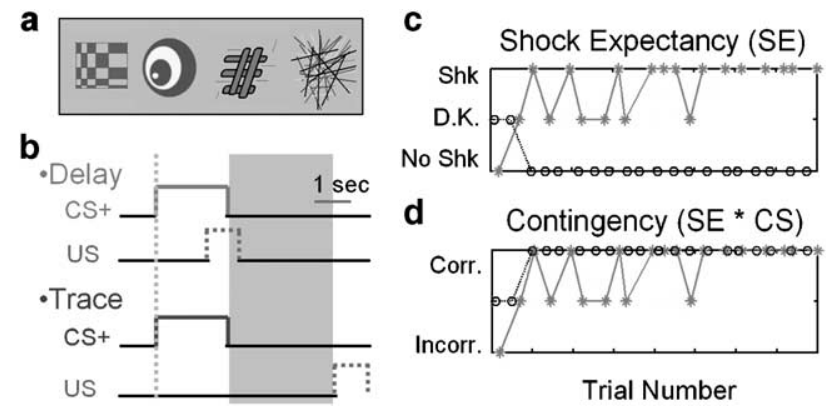

Fig. 1. Experimental design - 14 subjects reported shock expectancy during concurrent delay and trace aversive conditioning while functional brain images were acquired. Each CS presentation was $2 \mathrm{~s}$. Reinforced CS+ trials were followed by an electric shock lasting $1 \mathrm{~s}$. For each subject, the four images in panel a (presented in color) acted as either the delay CS+ (image presentation overlaps with the shock, see panel b), trace CS+ (image presentation ends $3 \mathrm{~s}$ before the onset of the shock, see panel $\mathrm{b}$ ), or as one of two CS - baselines. The point used for modeling event-related analysis for each trial is marked with a dotted line in panel b. The memory trace period, $3 \mathrm{~s}$ marked by a shaded box (also in panel b), was analyzed separately and is not discussed here. As soon as each image appeared, subjects had to judge the likelihood of being shocked (shock expectancy; c) using one of three keys indicating "shock likely", "don't know", or "no shock likely" (* $=\mathrm{CS}+$; $\mathrm{O}=\mathrm{CS}-)$. (d) The accuracy of contingency awareness is the interaction between shock expectancy (SE) and CS+/$(*=\mathrm{CS}+; \mathrm{O}=\mathrm{CS}-)$. This measure reflects when a subject provided an accurate report of the CS/US contingency over the course of the experiment, and defines the measure of accuracy of explicit knowledge. type, precluding study of the time course of learning. The remaining subjects are reported in the analysis: 9 male and 5 female, age range 19-31 (mean 24.7). All subjects gave prior informed consent. This study was approved by the Joint Ethics Committee of the National Hospital for Neurology and Neurosurgery, UK (UCLH NHS Trust) and the Internal Review Board at the California Institute of Technology, USA.

\section{Experimental procedure}

We performed concurrent trace and delay Pavlovian conditioning. The CSs were abstract colored images (see Fig. 1a) presented for $2 \mathrm{~s}$ and the US was a $1 \mathrm{~s}$ electrical stimulus (see below). The study comprised 160 individual trials involving four separate CSs (each presented 40 times). One of the images acted as the trace conditioning cue (trace CS), which was followed on $50 \%$ of occasions by the US after a $3 \mathrm{~s}$ trace interval. Another image acted as the delay conditioning cue (delay CS), followed on $50 \%$ of occasions by the US, with a $0.5 \mathrm{~s}$ overlap between the end of the CS and the start of the US. The remaining two images acted as neutral cues (CS-), never followed by the US. Images were counter-balanced across conditions between subjects. Presentations of the CSs were arranged randomly, such that two of each CS type appeared in a block of eight. The delay and trace CS were each reinforced once in every block of eight trials. Trials were triggered on the nearest slice using a pseudo-randomized inter-trial onset asynchrony of $8,9.25,10.5$, or $12 \mathrm{~s}$. Presentation of stimuli and timing were controlled using Cogent 2000 (Wellcome Department of Imaging Neuroscience, Institute of Neurology, London, UK).

\section{Online subject reports of contingency awareness}

Subjects reported US (electric shock) expectancy for each trial by pressing one of three keys with their right hand. This was performed as quickly as possible following the presentation of each CS. One key indicated that a shock was expected, the second key indicated that the subject did not know whether or not a shock was expected, and the third indicated that no shock was expected. Prior to conditioning, subjects practiced the procedure on a set of abstract images not used in the experiment. At no point before the experiment were participants explicitly informed about any relationship between the images (CSs) and shock (US). Failures to respond or responses where latencies exceeded $1.5 \mathrm{~s}$ were scored as "don't know".

\section{Post-experimental questionnaire}

Following scanning, subjects were given a post-experimental questionnaire similar to that used by Clark and Squire (1998). The questionnaire assessed their knowledge of the CS/US contingency relationships for both delay and trace protocols (see Supplementary material). Subjects rated each statement on a 7 point scale ranging from "not true" through "don't know" to "true" capturing their degree of confidence. A response that was both accurate and very confident received a score of +3 and a response that was inaccurate and very confident received a score of -3 with all other responses falling on a scale between these limits. Scores for each subject were then totaled, giving a potential range of -48 to +48 for each protocol. More positive scores reflect greater contingency awareness. 


\section{Unconditioned stimuli}

The pain specific shock was delivered to the top of the right foot using a $100 \mathrm{~Hz}$ train of square-waveform electrical pulses for $1 \mathrm{~s}$, via a bipolar concentric surface electrode (stimulation area $20 \mathrm{~mm}^{2}$ ) which selectively depolarizes A delta fibers (Kaube et al., 2000). This custom built concentric electrode was designed to limit activation to fast acting fibers and to reduce any possibility of muscle stimulation. The electrical stimulus was delivered via an optically isolated unit with a range of 0-12 mA. Current levels were chosen for each subject before the experiment, starting at a low level and using an ascending rating method where the current amplitude was raised until the subject gave a rating of 9 on a scale of $1-10$, where 1 indicated that the subject could barely feel the shock and 10 indicated that the shock was too uncomfortable to be used in the experiment.

\section{$S C R$}

Skin conductance data were collected at a minimum of $100 \mathrm{~Hz}$, and was aligned to the first slice pulse where scanning had started. Data collected at a rate higher than $100 \mathrm{~Hz}$ were first down-sampled to that frequency. Before analysis, all skin conductance data were median filtered to reduce noise. Skin conductance responses (SCRs) were defined as the maximum amplitude response initiated no earlier than $1 \mathrm{~s}$ with a peak no later than $5 \mathrm{~s}$ after the CS onset. SCR amplitudes were range corrected by the maximum response for that subject. A two-tailed, single sample $t$ test across subjects $(n=14)$ showed a significant difference between the mean non-reinforced $\mathrm{CS}+$ response and the mean $\mathrm{CS}-$ response for both delay $(P<0.01)$ and trace $(P<0.01)$ conditioning.

\section{$f M R I$}

Forty-four slice, whole brain, tilted axial BOLD images were acquired in a 3 T Siemens Allegra scanner using a gradient-echo EPI sequence (Deichmann et al., 2003), at a within plane resolution of $3 \mathrm{~mm}$ (TR $=2.86 \mathrm{~s})$. A total of 565 images were acquired, including five saturation scans which were later discarded. After the completion of the experiment, a T1 weighted anatomical scan was obtained for each subject.

Functional image analysis was performed using SPM2 (Wellcome Department of Imaging Neuroscience, Institute of Neurology, London, UK). Prior to analysis, all functional images were realigned, slice time acquisition corrected, normalized to the MNI EPI template, and smoothed using a Gaussian kernel ( $8 \mathrm{~mm}$ FWHM including contrast image smoothing). Individual subject models were then constructed and random effects analysis conducted as noted below. Each trial was modeled in two segments: first, an initial event-related response to each CS presentation and second, a $3 \mathrm{~s}$ period following image termination. This was done for both trace and delay conditioning to ensure that correlations between the stimulus event and trace period models were treated similarly. Trace period regressors were orthogonalized with respect to their eventrelated $\mathrm{CS}$ onset equivalents to minimize any contamination of the trace period response by the CS onset response. Only results for the CS onset event responses are reported here.

The acquisitions of both conditioning and contingency awareness were modeled as a parametric modulation (Buchel et al., 1998b) of responses to a CS presentation (indexed by skin conductance and US expectancy, respectively). In testing for brain activity that correlated with learning, we examined BOLD responses specific to the CS by performing a conjunction analysis (Friston et al., 1999). This technique identifies only those regions which show significant activation across the included conditions. Statistically, a conjunction analysis is identical to performing an $F$ test with the constraint that the individual effects are positive. For all reported results, we identified regions whose activity reflected learning for both delay and trace conditioning during all CS + trials (reinforced and non-reinforced). This provides a general measure of learned response differences specific to a CS presentation, leaving aside effects due to the presence or absence of a shock and those due to peculiarities of the specific conditioning protocol. A region is reported as being active in this conjunction if it shows a statistically significant violation of the null hypothesis that on average, members of the conjunction showed no effect (global null). An identified region's consistent activation for all members of the conjunction is confirmed by looking at the least significant $P$ value for any member of that conjunction. We include plots of correlations in Fig. 2 to demonstrate the consistency of a given effect across conditions included in the conjunction. This procedure lessens response ambiguity due to either the presence or absence of a US or any differences in protocol. Using a conjunction across these conditions allows us to infer a network that relates to the overall learned differences between $\mathrm{CS}+$ (delay and trace) and CS(neutral) representations. In areas where there was a prior hypothesis, results were family wise error (FWE) corrected for multiple comparisons using small volume correction $(20 \mathrm{~mm}$ diameter sphere centered at the peak of activation). FWE correction for multiple comparisons for the whole brain is applied to brain regions where there was no prior hypothesis.

\section{Learning: contingency awareness and conditioning}

Explicit learning accuracy was defined by the interaction between $\mathrm{CS}$ type (CS+ or $\mathrm{CS}-$ ) and the reported US expectancy for each trial. Brain activity that correlated with US expectancy alone would have corresponded to those areas relevant for explicit fear, whereas shock expectancy by CS type $(+$ or -$)$ interaction tests for brain activity that correlates with the accuracy of shock expectancy on each trial. The magnitude of explicit learning defined by the subject's score on the post-experimental questionnaire was used as a subject covariate in a second level random effects analysis (Fig. 2 and Table 1b).

Implicit learning accuracy was defined by the interaction between CS type (+ or -) and the normalized amplitude of the skin conductance response for each trial. The magnitude of implicit learning was defined as the average difference between $\mathrm{CS}+$ and $\mathrm{CS}$ - skin conductance responses and was used as a subject covariate in a second level random effects analysis (Table 1a).

\section{Results}

\section{Conditioned skin conductance responses}

We recorded skin conductance responses associated with visual cues to provide an implicit measure of conditioning. Activity in the left amygdala $(-27,-3,-12)$ correlated with the trial-by-trial time course of conditioning, indexed by the level of discriminatory skin conductance responses $(P<0.01$ corrected, see Methods and Table 1a). This result confirms previous 

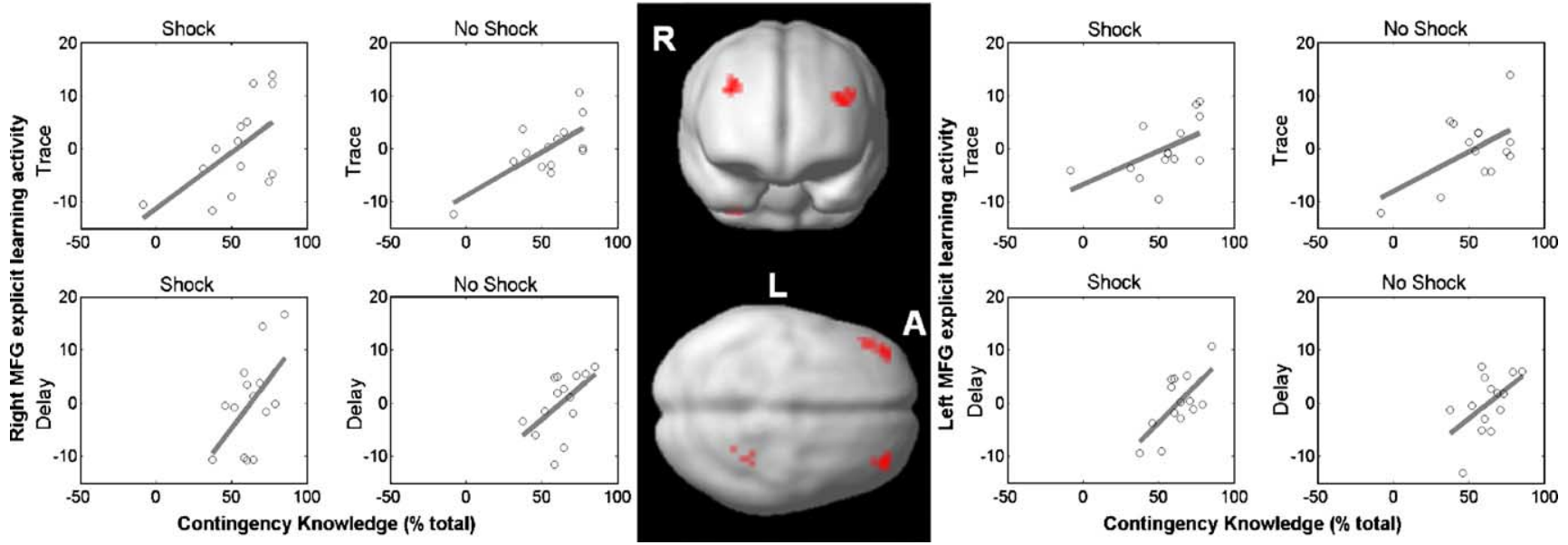

Fig. 2. Activity in the middle frontal gyrus correlates with explicit learning. The center image shows a surface rendering of bilateral middle frontal gyri activity that correlates with explicit learning. Middle frontal gyrus activity is consistently correlated with explicit learning for all conditions reflecting its general role in the acquisition of accurate explicit knowledge. Regression plots of brain activity (the explicit learning time course parameter estimate) vs. contingency knowledge (post experimental questionnaire score) are shown for the right and left middle frontal gyrus for trace and delay protocols during shock reinforced $\mathrm{CS}+$, and no shock CS+ trials. A contingency knowledge score of $100 \%$ indicates that the subject was able to answer every question about the exact temporal relationship between CS and US accurately with high confidence. $0 \%$ is chance performance.

findings (Buchel et al., 1998a, 1999; Knight et al., 2004) and in addition demonstrates that amygdala activity correlates with the specific time course of learning and incorporates the relative success of conditioning in different subjects.

\section{Contingency awareness}

Our principle goal was to identify neural responses correlating with contingency awareness, an example of explicit or

Table 1

Brain regions whose BOLD responses correlate with implicit and explicit measures of learning are shown in (a) and (b)

\begin{tabular}{|c|c|c|c|c|}
\hline Cluster Region & MNI coordinates & Voxels & $\begin{array}{l}P \text { value } \\
\text { (L.S.) }\end{array}$ & $\begin{array}{l}P \text { value } \\
\text { (G.N.) }\end{array}$ \\
\hline \multicolumn{5}{|l|}{ (a) Implicit learning } \\
\hline $\begin{array}{l}\text { Left hippocampus/ } \\
\text { subiculum }\end{array}$ & $(-12,-30,-6)$ & 364 & 0.05 & $P<0.01^{\mathrm{a}}$ \\
\hline $\begin{array}{l}\text { Right hippocampus/ } \\
\text { subiculum }\end{array}$ & $(21,-27,-12)$ & & 0.04 & $P<0.01^{\mathrm{b}}$ \\
\hline $\begin{array}{l}\text { Occipital cortex } \\
\text { (posterior pole) }\end{array}$ & $(9,-102,-9)$ & 34 & 0.02 & $P<0.01^{\mathrm{b}}$ \\
\hline Left amygdala & $(-27,-3,-12)$ & 11 & 0.06 & $P<0.01^{\mathrm{a}}$ \\
\hline \multicolumn{5}{|l|}{ (b) Explicit learning } \\
\hline $\begin{array}{l}\text { Left middle } \\
\text { frontal gyrus }\end{array}$ & $(-36,51,30)$ & 35 & 0.02 & $P<0.001^{\mathrm{a}}$ \\
\hline $\begin{array}{l}\text { Right middle } \\
\text { frontal gyrus }\end{array}$ & $(36,51,36)$ & 22 & 0.04 & $P<0.01^{\mathrm{a}}$ \\
\hline $\begin{array}{l}\text { Left parahippocampal } \\
\text { gyrus }\end{array}$ & $(-15,-15,-24)$ & 3 & 0.096 & $P=0.55$ \\
\hline
\end{tabular}

This table specifies the anatomical labels for responsive clusters with the location of peak significance ( $\mathrm{mm}$ in MNI space), the number of voxels included in the cluster (threshold $=P<0.001$ ), and the global null (effects of interest) $P$ value for the peak voxel in each cluster (G.N.). To ensure the consistency of correlation across conditions, the least significant individual $P$ value (L.S.) is also reported.

${ }^{\text {a }}$ FWE corrected for small volume (20 $\mathrm{mm}$ diameter sphere).

${ }^{b}$ FWE corrected for whole brain. declarative learning, measured by both online reports and postexperimental questionnaire. Online reports assessed US expectancy on a trial-by-trial basis: expectancy was accurate when it was high for a $\mathrm{CS}+$ (predicts the shock) presentation and low for a CS- (neutral) presentation. This relationship is described as the accuracy of contingency awareness (Fig. 1d). The magnitude of a subject's contingency awareness was defined by a postexperimental questionnaire score which assessed the individual's overall contingency knowledge via a series of true/false statements about the CS/US relationship (see Methods and Supplementary materials).

Brain regions that correlated with contingency awareness had greater activity during trials where a subject accurately expects a shock. We accounted for inter-subject differences by testing for regions that showed greater activity in those subjects who scored higher on the post-experimental questionnaire. This revealed responses correlated with contingency awareness in bilateral middle frontal gyri (MFG) (Fig. 2, Table 1b, left -36, 51, 30; right $36,51,36)$, significant after correction for multiple comparisons. We also noted correlated activity in the parahippocampal gyrus $(-15,-15,-24, P=0.055$ corrected for multiple comparisons, see Methods).

\section{Discussion}

Our data indicate a clear role for the middle frontal gyrus in contingency awareness during conditioning, correlated specifically with the acquisition of awareness on a trial-by-trial basis. To our knowledge, this is the first time such a trial-by-trial link has been demonstrated during conditioning. The role of the middle frontal gyrus in contingency awareness is contrasted with involvement of the amygdala which we show to reflect the acquisition of implicit knowledge, as indexed by autonomic activity, consistent with previous research (Buchel et al., 1998a, 1999; LeDoux, 2003). These results clearly dissociate the distinct roles of the middle frontal gyrus and amygdala during classical conditioning. 
In delay eye-blink conditioning, the magnitude of conditioning is independent of explicit knowledge (Manns et al., 2002). However, explicit learning is likely to be expressed in delay protocols, even if it is not correlated with the degree of conditioning. Since the degree of implicit knowledge is not correlated with the degree of explicit knowledge in delay conditioning, the substrates mediating both forms of learning can be separated. We confirmed that those same neural substrates were active during trace conditioning by testing for areas whose activation was consistent across both delay and trace conditioning. The fact that the middle frontal gyrus is active in both trace and delay conditioning, even though trace conditioning is correlated with contingency knowledge and delay is not (Clark and Squire, 1998), has implications for the mechanism contingency knowledge facilitates conditioning. The middle frontal gyrus is unlikely to directly facilitate conditioned associations, since doing so would require a second inhibitory mechanism active during delay conditioning. It is therefore more likely that the middle frontal gyrus facilitates conditioning by means of another brain area, such as the hippocampal complex (see below). While it is unlikely that prefrontal areas directly facilitate conditioning, it is important to keep in mind that there is evidence that areas of prefrontal cortex inhibit activity in the amygdala (Rosenkranz and Grace, 2001; Quirk et al., 2003).

An area homologous to the middle frontal gyrus, the medial prefrontal cortex in the rabbit, is necessary for trace eye-blink conditioning (Kronforst-Collins and Disterhoft, 1998). This region is also strongly implicated in tasks requiring maintenance and manipulation of information within working memory in humans (D’Esposito et al., 1998; Leung et al., 2002; Pessoa et al., 2002) and in animal models of working memory (Goldman-Rakic, 1987; Petrides, 2000; Castner et al., 2004). We have previously shown that working memory distraction during fear conditioning reduces explicit knowledge of the CS/US contingency (Carter et al., 2003). This reduction of explicit knowledge is consistent with the middle frontal gyri's involvement in both working memory and contingency awareness.

Brain areas central to the expression of explicit knowledge, as required in reporting contingencies, may play a role in abstract, symbolic manipulation. In line with this notion, neurons in the middle frontal gyrus of behaving macaque monkeys respond to specific rules (Wallis et al., 2001) or limit responses to a given stimulus to only those times when a specific practiced task is being performed (Asaad et al., 2000). Thus, our finding that activity in this region correlates with contingency awareness is consistent with a putative role in the representation of abstract concepts.

We also found activity in the left parahippocampal gyrus correlated with contingency awareness during conditioning. These results point to a role for the hippocampal complex in mediating the integration of explicit knowledge of contingencies (Eichenbaum et al., 1996; Clark and Squire, 1998). It is interesting that while we found significant contingency related activation in the parahippocampal gyrus, we did not find such effects in the hippocampus proper. By contrast, we observed a significant correlation between activity in the hippocampus proper and our implicit measure of conditioning. These results do suggest the intriguing possibility that different sub-regions of hippocampal complex have dissociable roles in associative learning, in line with evidence that the hippocampus is involved in the integration of cues and not simply related to explicit knowledge (Chun and Phelps, 1999; Schendan et al., 2003;
Degonda et al., 2005). Future studies will need to address the mechanism of integration.

In conclusion, our study provides new evidence that the trialby-trial accuracy of contingency knowledge during conditioning involves the middle frontal gyri and a sub-region of the hippocampal complex, across both delay and trace conditioning. These findings give insight not only into the neural substrates of classical trace protocols, where explicit knowledge correlates with conditioning, but suggest a substrate for how explicit knowledge is coded in the human brain.

\section{Acknowledgments}

This work was supported by grants from the Gordon Moore Foundation, the National Science Foundation, Sandia National Laboratory, and the Wellcome Trust Programme Grant to RJD. RMC would like to thank N. Tsuchiya, C. Hofstötter, and K. Watson for discussions and comments on the manuscript.

\section{Appendix A. Supplementary data}

Supplementary data associated with this article can be found in the online version at doi:10.1016/j.neuroimage.2005.09.011.

\section{References}

Asaad, W.F., Rainer, G., Miller, E.K., 2000. Task-specific neural activity in the primate prefrontal cortex. J. Neurophysiol. 84, 451-459.

Bechara, A., Tranel, D., Damasio, H., Adolphs, R., Rockland, C., Damasio, A.R., 1995. Double dissociation of conditioning and declarative knowledge relative to the amygdala and hippocampus in humans. Science 269, 1115-1118.

Buchel, C., Dolan, R.J., 2000. Classical fear conditioning in functional neuroimaging. Curr. Opin. Neurobiol. 10, 219-223.

Buchel, C., Morris, J., Dolan, R.J., Friston, K.J., 1998a. Brain systems mediating aversive conditioning: an event-related fMRI study. Neuron 20, 947-957.

Buchel, C., Holmes, A.P., Rees, G., Friston, K.J., 1998b. Characterizing stimulus-response functions using nonlinear regressors in parametric fMRI experiments. NeuroImage 8, 140-148.

Buchel, C., Dolan, R.J., Armony, J.L., Friston, K.J., 1999. Amygdalahippocampal involvement in human aversive trace conditioning revealed through event-related functional magnetic resonance imaging. J. Neurosci. 19, 10869-10876.

Carter, R.M., Hofstotter, C., Tsuchiya, N., Koch, C., 2003. Working memory and fear conditioning. Proc. Natl. Acad. Sci. U. S. A. 100, $1399-1404$.

Castner, S.A., Goldman-Rakic, P.S., Williams, G.V., 2004. Animal models of working memory: insights for targeting cognitive dysfunction in schizophrenia. Psychopharmacology (Berlin) 174, $111-125$.

Chun, M.M., Phelps, E.A., 1999. Memory deficits for implicit contextual information in amnesic subjects with hippocampal damage. Nat. Neurosci. 2, 844-847.

Clark, R.E., Squire, L.R., 1998. Classical conditioning and brain systems: the role of awareness. Science 280, 77-81.

Clark, R.E., Manns, J.R., Squire, L.R., 2002. Classical conditioning, awareness, and brain systems. Trends Cogn. Sci. 6, 524-531.

Cole, L.E., 1939. A comparison of factors of practice and knowledge of experimental procedure in conditioning the eye-lid response of human subjects. J. Gen. Psychol. 20, 349-373. 
Colloca, L., Benedetti, F., 2005. Placebos and painkillers: is mind as real as matter? Nat. Rev., Neurosci. 6, 545-552.

Compton, D.M., Griffith, H.R., McDaniel, W.F., Foster, R.A., Davis, B.K., 1997. The flexible use of multiple cue relationships in spatial navigation: a comparison of water maze performance following hippocampal, medial septal, prefrontal cortex, or posterior parietal cortex lesions. Neurobiol. Learn. Mem. 68, 117-132.

Cook, S.W., Harris, R.E., 1937. The verbal conditioning of the galvanic skin response. J. Exp. Psychol. 21, 202-210.

Corlett, P.R., Aitken, M.R., Dickinson, A., Shanks, D.R., Honey, G.D., Honey, R.A., Robbins, T.W., Bullmore, E.T., Fletcher, P.C., 2004. Prediction error during retrospective revaluation of causal associations in humans: fMRI evidence in favor of an associative model of learning. Neuron 44, 877-888.

Dawson, M.E., Furedy, J.J., 1976. The role of awareness in human differential autonomic classical conditioning: the necessary-gate hypothesis. Psychophysiology 13, 50-53.

Degonda, N., Mondadori, C.R., Bosshardt, S., Schmidt, C.F., Boesiger, P., Nitsch, R.M., Hock, C., Henke, K., 2005. Implicit associative learning engages the hippocampus and interacts with explicit associative learning. Neuron 46, 505-520.

Deichmann, R., Gottfried, J.A., Hutton, C., Turner, R., 2003. Optimized EPI for fMRI studies of the orbitofrontal cortex. NeuroImage 19, $430-441$.

D’Esposito, M., Aguirre, G.K., Zarahn, E., Ballard, D., Shin, R.K., Lease, J., 1998. Functional MRI studies of spatial and nonspatial working memory. Cogn. Brain Res. 7, 1-13.

Eichenbaum, H., Schoenbaum, G., Young, B., Bunsey, M., 1996. Functional organization of the hippocampal memory system. Proc. Natl. Acad. Sci. U. S. A. 93, 13500-13507.

Fanselow, M.S., 2000. Contextual fear, gestalt memories, and the hippocampus. Behav. Brain Res. 110, 73-81.

Friston, K.J., Holmes, A.P., Price, C.J., Buchel, C., Worsley, K.J., 1999. Multisubject fMRI studies and conjunction analyses. NeuroImage 10, $385-396$.

Goldman-Rakic, P., 1987. Circuitry of primate prefrontal cortex and regulation of behaviour by representational memory. In: Plum, F., Mouncastle, U. (Eds.), Handbook of Physiology. The American Physiological Society, Washington, DC, pp. 373-417.

Grillon, C., 2002. Startle reactivity and anxiety disorders: aversive conditioning, context, and neurobiology. Biol. Psychiatry 52, 958-975.

Han, C.J., O'Tuathaigh, C.M., van Trigt, L., Quinn, J.J., Fanselow, M.S., Mongeau, R., Koch, C., Anderson, D.J., 2003. Trace but not delay fear conditioning requires attention and the anterior cingulate cortex. Proc. Natl. Acad. Sci. U. S. A. 100, 13087-13092.

Hilgard, E.R., Campbell, R.K., Sears, W.N., 1937. Conditioned discrimination: the effect of knowledge of stimulus relationships. Am. J. Psychol. 51, 498-506.

Kaube, H., Katsarava, Z., Kaufer, T., Diener, H.C., Ellrich, J., 2000. A new method to increase nociception specificity of the human blink reflex. Clin. Neurophysiol. 111, 413-416.

Knight, D.C., Cheng, D.T., Smith, C.N., Stein, E.A., Helmstetter, F.J., 2004. Neural substrates mediating human delay and trace fear conditioning. J. Neurosci. 24, 218-228.

Knuttinen, M.G., Power, J.M., Preston, A.R., Disterhoft, J.F., 2001.
Awareness in classical differential eyeblink conditioning in young and aging humans. Behav. Neurosci. 115, 747-757.

Kronforst-Collins, M.A., Disterhoft, J.F., 1998. Lesions of the caudal area of rabbit medial prefrontal cortex impair trace eyeblink conditioning. Neurobiol. Learn. Mem. 69, 147-162.

LeDoux, J., 2003. The emotional brain, fear, and the amygdala. Cell. Mol. Neurobiol. 23, 727-738.

Leung, H.C., Gore, J.C., Goldman-Rakic, P.S., 2002. Sustained mnemonic response in the human middle frontal gyrus during on-line storage of spatial memoranda. J. Cogn. Neurosci. 14, 659-671.

Lovibond, P.F., Shanks, D.R., 2002. The role of awareness in Pavlovian conditioning: empirical evidence and theoretical implications. J. Exp. Psychol. Anim. Behav. Processes 28, 3-26.

Manns, J.R., Clark, R.E., Squire, L.R., 2002. Standard delay eyeblink classical conditioning is independent of awareness. J. Exp. Psychol. Anim. Behav. Processes 28, 32-37.

Maren, S., 2001. Neurobiology of Pavlovian fear conditioning. Annu. Rev. Neurosci. 24, 897-931.

Maren, S., Quirk, G.J., 2004. Neuronal signalling of fear memory. Nat. Rev., Neurosci. 5, 844-852.

McEchron, M.D., Bouwmeester, H., Tseng, W., Weiss, C., Disterhoft, J.F., 1998. Hippocampectomy disrupts auditory trace fear conditioning and contextual fear conditioning in the rat. Hippocampus 8, 638-646.

Ohman, A., Soares, J.J., 1998. Emotional conditioning to masked stimuli: expectancies for aversive outcomes following nonrecognized fearrelevant stimuli. J. Exp. Psychol. Gen. 127, 69-82.

Olsson, A., Phelps, E.A., 2004. Learned fear of "unseen" faces after Pavlovian, observational, and instructed fear. Psychol. Sci. 15, $822-828$.

Pavlov, I.P., 1906. The scientific investigation of the psychical faculties or processes in higher animals. Science 24, 613-619.

Pessoa, L., Gutierrez, E., Bandettini, P., Ungerleider, L., 2002. Neural correlates of visual working memory: fMRI amplitude predicts task performance. Neuron 35, 975-987.

Petrides, M., 2000. The role of the mid-dorsolateral prefrontal cortex in working memory. Exp. Brain Res. 133, 44-54.

Quirk, G.J., Gehlert, D.R., 2003. Inhibition of the amygdala: key to pathological states? Ann. N. Y. Acad. Sci. 985, 263-272.

Quirk, G.J., Likhtik, E., Pelletier, J.G., Pare, D., 2003. Stimulation of medial prefrontal cortex decreases the responsiveness of central amygdala output neurons. J. Neurosci. 23, 8800-8807.

Rosenkranz, J.A., Grace, A.A., 2001. Dopamine attenuates prefrontal cortical suppression of sensory inputs to the basolateral amygdala of rats. J. Neurosci. 21, 4090-4103.

Schendan, H.E., Searl, M.M., Melrose, R.J., Stern, C.E., 2003. An FMRI study of the role of the medial temporal lobe in implicit and explicit sequence learning. Neuron 37, 1013-1025.

Wallis, J.D., Anderson, K.C., Miller, E.K., 2001. Single neurons in prefrontal cortex encode abstract rules. Nature 411, 953-956.

Wiens, S., Ohman, A., 2002. Unawareness is more than a chance event: comment on Lovibfcond and Shanks 2002. J. Exp. Psychol. Anim. Behav. Processes 28, 27-31.

Wilensky, A.E., Schafe, G.E., LeDoux, J.E., 1999. Functional inactivation of the amygdala before but not after auditory fear conditioning prevents memory formation. J. Neurosci. 19, 1-5 (art. no.-RC48). 